\title{
A PROOF OF THE VERACITY OF BOTH GOLDBACH AND DE POLIGNAC CONJECTURES
}

\author{
Jamel Ghanouchi \\ 6 Rue Khansa 2070 Marsa Tunisie
}

Keywords : Goldbach ; De Polignac ; Twin primes ; Algebraic proof

\section{Abstract}

The present algebraic development begins by an exposition of the data of the problem. The definition of the primal radius $r>0$ is : For all positive integer $x \geq 3$ exists a fi nte nu or of integers called the primal radius $r>0$, for which $x+r$ and $x-r$ are prime numb The con ary is that $2 x=(x+r)+(x-r)$ is always the sum of a finite number of primes. 1so, all po tive integer $x \geq 0$, exists an infinity of integers $r>0$, for which $x+r$ and $r-y$ are prime i ber. The conclusion is that $2 x=(x+r)-(r-x)$ is always an infinity of difference prim

\section{Introduction}

There is a similarity between the assertion : "an even number alwa he sum of two primes" and the assertion: "an even number is always the difference pros primes the prest article gives the proof that the two assertions are the consequences of the same concept oy the introduction of the notion of the primal radius.

\section{The proof}

Let us suppose that exists an integer $x \geq 3$ for $\mathrm{n}$ ich $p_{1}$ and $p_{2}$ primes, $\left.3 \leq p_{2}<p_{1}, 2 x=p_{1}+\right)$ or $2 x=p_{1}+p_{2}+2 b_{p_{1}, p_{2}}=p_{1}+p_{2}+2 b$
then $x=\frac{p_{1}+p_{2}}{2}+b$.
But for all $p_{1}, p_{2}$ exist

Let

$x_{1}=p_{1}+2 b, x_{2}=-2 b, x_{1}=p_{2}+2 b, x_{4}=p_{1}-2 b$

We deduce tha

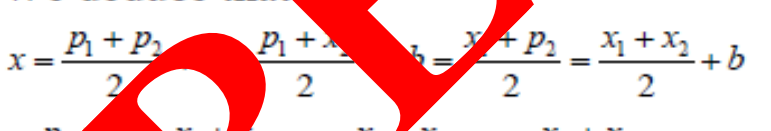

$=\frac{p_{1}}{2}-\frac{x_{1}}{2}+\frac{x_{3}}{2}+b=\frac{x_{4}+x_{2}}{2}+3 b$

$y=\frac{p_{1}-p_{2}}{2}+b \frac{n_{1}-x_{2}}{2}=\frac{x_{1}-p_{2}}{2}=\frac{x_{1}-x_{2}}{2}-b$

$=\frac{p_{1}-x_{3}}{2}+2 b=\frac{x_{1}-x_{3}}{2}+b=\frac{x_{4}-x_{3}}{2}+3 b=\frac{x_{4}-x_{2}}{2}+b$

$x_{1}+x_{2}=p_{1}+p_{2}$

$x_{1}-x_{3}=p_{1}-p_{2}$ 


\section{Lemma 1}

The following formula

$$
\begin{aligned}
& x=\frac{p_{1}+p_{2}}{2}+b=\frac{p_{1}+x_{2}}{2}+2 b=\frac{x_{1}+p_{2}}{2}=\frac{x_{1}+x_{2}}{2}+b \\
& =\frac{p_{1}+x_{3}}{2}=\frac{x_{1}+x_{3}}{2}-b=\frac{x_{4}+x_{3}}{2}+b=\frac{x_{4}+x_{2}}{2}+3 b \\
& y=\frac{p_{1}-p_{2}}{2}+b=\frac{p_{1}-x_{2}}{2}=\frac{x_{1}-p_{2}}{2}=\frac{x_{1}-x_{2}}{2}-b \\
& =\frac{p_{1}-x_{3}}{2}+2 b=\frac{x_{1}-x_{3}}{2}+b=\frac{x_{4}-x_{3}}{2}+3 b=\frac{x_{4}-x_{2}}{2}+b
\end{aligned}
$$

Imply that exist $p_{1}$ and $p_{2}$ prime numbers for which $b=0$

\section{Proof of lemma 1}

If $x$ is prime $2 \mathrm{x}=\mathrm{x}+\mathrm{x}$ is the sum of two primes, then $\mathrm{p} 1-\mathrm{p} 2 \neq 0$

We will suppose firstly that $\left(x_{1}-x_{2}\right)\left(x_{1}+x_{3}\right) \neq 0$

Let

$\frac{x_{1}-x_{2}}{p_{1}-p_{2}}=\frac{p_{1}-p_{2}+4 b}{p_{1}-p_{2}}=1+\frac{4 b}{p_{1}-p_{2}}$

$\frac{p_{1}-p_{2}}{x_{1}-x_{2}}=\frac{x_{1}-x_{2}-4 b}{x_{1}-x_{2}}=1-\frac{4 b}{x_{1}-x_{2}}$

We pose $k=\frac{2 b}{p_{1}-p_{2}}, k^{\prime}=-\frac{2 b}{x_{1}-x_{2}}$

$k k^{\prime}=0 \Rightarrow b=0$, we have suppose

$\forall(x, y) ; \exists \varphi \mid x=\varphi y$

$x+y=(\varphi+1) y=x_{1} \neq 0, x-y$

$\forall(k, k) ; \exists \alpha \mid k=\alpha k^{\prime}$

$\Rightarrow \frac{2 b}{p_{1}-p_{2}}=-\alpha \frac{2 b}{x_{1}-x}$

$\Rightarrow x_{1}-x_{2}=-\alpha\left(p, \quad p_{2}\right) \Rightarrow-x_{2}-p_{1}+\rho_{2}=4 b=-(\alpha+1)\left(p_{1}-p_{2}\right)$

$\Rightarrow b=-\frac{\alpha+1}{4}\left(p_{1}-2\right.$

$\Rightarrow x=y+\frac{1+p_{2}}{2}, b=\frac{p_{1}+\lambda}{2} \quad \frac{\alpha+1}{4}\left(p_{1}-p_{2}\right)=\frac{(1-\alpha) p_{1}+(3+\alpha) p_{2}}{4}=\frac{\varphi}{\varphi-1} p_{2}$

$y=\frac{p_{1}-p_{2}}{2}=\frac{p_{1}-p_{2}}{2}-\frac{\alpha+1}{4}\left(p_{1}-p_{2}\right)=\frac{(1-\alpha)\left(p_{1}-p_{2}\right)}{4}=\frac{1}{\varphi-1} p_{2}$

Let

$$
\begin{aligned}
& \frac{x_{1}+x_{3}}{p_{1}+p_{2}}=\frac{p_{1}+p_{2}+4 b}{p_{1}+p_{2}}=1+\frac{4 b}{p_{1}+p_{2}} \\
& \frac{p_{1}+p_{2}}{x_{1}+x_{3}}=\frac{x_{1}+x_{3}-4 b}{x_{1}+x_{3}}=1-\frac{4 b}{x_{1}+x_{3}}
\end{aligned}
$$


We pose $\quad m=\frac{2 b}{p_{1}+p_{2}}, m^{\prime}=-\frac{2 b}{x_{1}+x_{3}}$

$m m^{\prime}=0 \Rightarrow b=0$, we have supposed $m m^{\prime} \neq 0$

$\forall(m, m) ; \exists \beta \mid m=\beta m^{\prime}$

$$
\begin{aligned}
& \Rightarrow \frac{2 b}{p_{1}+p_{2}}=-\beta \frac{2 b}{x_{1}+x_{3}} \\
& \Rightarrow x_{1}+x_{3}=-\beta\left(p_{1}+p_{2}\right) \Rightarrow x_{1}+x_{3}-p_{1}-p_{2}=4 b=-(\beta+1)\left(p_{1}+p_{2}\right) \\
& \Rightarrow b=-\frac{\beta+1}{4}\left(p_{1}+p_{2}\right) \\
& \Rightarrow x=\frac{p_{1}+p_{2}}{2}+b=\frac{p_{1}+p_{2}}{2}-\frac{\beta+1}{4}\left(p_{1}+p_{2}\right)=\frac{(1-\beta)\left(p_{1}+p_{2}\right)}{4}=\frac{\varphi}{\varphi-1} p_{2} \\
& y=\frac{p_{1}-p_{2}}{2}+b=\frac{p_{1}-p_{2}}{2}-\frac{\beta+1}{4}\left(p_{1}+p_{2}\right)=\frac{(1-\beta) p_{1}-(\beta+3) p_{2}}{4}=\frac{1}{\varphi-1} p_{2} \\
& b=-\frac{\alpha+1}{4}\left(p_{1}-p_{2}\right)=-\frac{\beta+1}{4}\left(p_{1}+p_{2}\right) \Rightarrow(\beta-\alpha) p_{1}=(-2-\alpha-\beta) p_{2}
\end{aligned}
$$

But : $(2 k+1)\left(2 k^{\prime}+1\right)=1 \Rightarrow 2 k k^{\prime}+k+k^{\prime}=0 \Rightarrow(2 k+1) k^{\prime}=-k$

$2 k+1=\frac{-k}{k^{\prime}}=\frac{k+1}{k^{\prime}+1}=\frac{a(k+1)+a^{\prime}(2 k+1)}{a\left(k^{\prime}+1\right)+a^{\prime}}$

$2 k^{\prime}+1=\frac{-k^{\prime}}{k}=\frac{k^{\prime}+1}{k+1}=\frac{c\left(k^{\prime}+1\right)+c^{\prime}\left(2 k^{\prime}+1\right)}{c(k+1)+c^{\prime}}, \forall\left(a, a^{\prime}, c, c^{\prime}\right)$

$\frac{2 k+1}{2 k^{\prime}+1}=\frac{\left(\left(a+2 a^{\prime}\right) k+a+a^{\prime}\right)\left(c k+c+c^{\prime}\right)}{\left(\left(c+2 c^{\prime}\right) k^{\prime}+c+c^{\prime}\right)\left(a k^{\prime}+a+a^{\prime}\right)}$

$=\frac{\left(a c+2 a^{\prime} c\right) k^{2}+\left(2 a c+a c^{\prime}+3 a^{\prime} c+2 a^{\prime} c\right) k+a c+a c^{\prime}+a^{\prime} c+a^{\prime} c^{\prime}}{(a c+2 a c) k^{\prime 2}+\left(2 a c+3 a c^{\prime}+a^{\prime} c+2 a^{\prime} c\right) k^{\prime}+a c+a c^{\prime}+a^{\prime} c+a^{\prime} c^{\prime}}$

$\Rightarrow \frac{2 k+1}{2 k^{\prime}+1}-1=\left(k-k^{\prime}\right) \frac{2}{2 k^{\prime}+1}$

$=\frac{a c\left(k^{2}-k^{\prime 2}\right)+2 a^{\prime} c k^{2}-2 a c^{\prime} k^{\prime 2}+\left(2 a c+a c^{\prime}+a^{\prime} c+2 a^{\prime} c^{\prime}\right) d-k^{\prime}+2 a}{(a c+2 a c) k^{\prime 2}+\left(2 a c+3 a c^{\prime}+a^{\prime} c+2\right.}$

$\forall\left(a, a^{\prime}, c, c^{\prime}\right)$, particularly $\left(a, a^{\prime}, c, c^{\prime}\right) \quad c^{\prime}=\delta k, a^{\prime} c=\delta k$

$\Rightarrow\left(k-k^{\prime}\right) \frac{2}{2 k^{\prime}+1}$

$\left.=\frac{a c(k+k)\left(k-k^{\prime}\right)+\left(2 a c+a c^{\prime}\right.}{(a c+2 a c) k^{\prime 2}+(2 a c+3}+2 a^{\prime} c^{\prime}\right)\left(k-a^{\prime} c \frac{2 \delta k k^{\prime 2}-2 \delta k^{2} k^{\prime}}{\left.c^{\prime}\right) k^{\prime}+a c+a c^{\prime}+a^{\prime} c+a^{\prime} c^{\prime}}\right.$

$=\left(k-k^{\prime}\right) \frac{-a c c x^{\prime}+2 a c+a c}{\left(a c+2 a c^{\prime} c^{\prime 2}+\left(2 a-3 a c^{\prime}+a^{\prime} c-2 a^{\prime} c^{\prime}\right) k^{\prime}+a c+a c^{\prime}+a^{\prime} c+a^{\prime} c^{\prime}\right.}$

If $\left.k=k^{\prime} \Rightarrow \alpha=1 \Rightarrow(1, \alpha) p_{1}-3+\alpha\right) p_{2}=p_{2}$, trivial solution : it is impossible.

$k-k^{\prime} \Rightarrow \frac{2 k}{2 k}=\frac{-2 a c k k^{\prime}+2 a c+a c^{\prime}+a^{\prime} c+2 a^{\prime} c^{\prime}-2 \delta k k^{\prime}}{\left(a c+2 c^{\prime}\right) k^{\prime 2}+\left(2 a c+3 a c^{\prime}+a^{\prime} c+2 a^{\prime} c^{\prime}\right) k^{\prime}+a c+a c^{\prime}+a^{\prime} c+a^{\prime} c^{\prime}}$

Also (2.) $\left(2 m+1,=1 \Rightarrow 2 m m^{\prime}+m+m^{\prime}=0 \Rightarrow(2 m+1) m^{\prime}=-m\right.$

$2 m+1=\frac{-m}{m^{\prime}}=\underset{m-1}{m-1}=\frac{a(m+1)+a^{\prime}(2 m+1)}{a\left(m^{\prime}+1\right)+a^{\prime}}$

$2 m^{\prime}+1=\frac{-m^{\prime}}{m}=\frac{m^{\prime}+1}{m+1}=\frac{c\left(m^{\prime}+1\right)+c^{\prime}\left(2 m^{\prime}+1\right)}{c(m+1)+c^{\prime}}, \forall\left(a, a^{\prime}, c, c^{\prime}\right)$

$\frac{2 m+1}{2 m^{\prime}+1}=\frac{\left((a+2 a) m+a+a^{\prime}\right)\left(c m+c+c^{\prime}\right)}{\left(\left(c+2 c^{\prime}\right) m^{\prime}+c+c^{\prime}\right)\left(a m^{\prime}+a+a^{\prime}\right)}$

$=\frac{\left(a c+2 a^{\prime} c\right) m^{2}+\left(2 a c+a c^{\prime}+3 a^{\prime} c+2 a^{\prime} c^{\prime}\right) m+a c+a c^{\prime}+a^{\prime} c+a^{\prime} c^{\prime}}{(a c+2 a c) m^{\prime 2}+\left(2 a c+3 a c^{\prime}+a^{\prime} c+2 a^{\prime} c^{\prime}\right) m^{\prime}+a c+a c^{\prime}+a^{\prime} c+a^{\prime} c^{\prime}}$

$\Rightarrow \frac{2 m+1}{2 m^{\prime}+1}-1=\left(m-m^{\prime}\right) \frac{2}{2 m^{\prime}+1}$ 
$=\frac{a c\left(m^{2}-m^{\prime 2}\right)+2 a^{\prime} m^{2}-2 a c^{\prime} m^{\prime 2}+\left(2 a c+a c^{\prime}+a^{\prime} c+2 a^{\prime} c^{\prime}\right)\left(m-m^{\prime}\right)+2 a^{\prime} c m-2 a c^{\prime} m^{\prime}}{\left(a c+2 a c^{\prime}\right) m^{\prime 2}+\left(2 a c+3 a c^{\prime}+a^{\prime} c+2 a^{\prime} c^{\prime}\right) m^{\prime}+a c+a c^{\prime}+a^{\prime} c+a^{\prime} c^{\prime}}$

$\forall\left(a, a^{\prime}, c, c^{\prime}\right)$, particularly $\left(a, a^{\prime}, c, c^{\prime}\right) \mid a c^{\prime}=\delta k^{2}=\gamma m^{2}, a^{\prime} c=\delta k^{\prime 2}=\gamma^{\prime} m^{\prime 2}$

$\Rightarrow\left(m-m^{\prime}\right) \frac{2}{2 m^{\prime}+1}$

$=\frac{a c\left(m+m^{\prime}\right)\left(m-m^{\prime}\right)+2\left(\gamma^{\prime}-\gamma\right) m^{2} m^{\prime 2}+\left(2 a c+a c^{\prime}+a^{\prime} c+2 a^{\prime} c^{\prime}\right)\left(m-m^{\prime}\right)+2 \gamma^{\prime} m m^{\prime 2}-2 \gamma m^{2} m^{\prime}}{\left(a c+2 a c^{\prime}\right) m^{\prime 2}+\left(2 a c+3 a c^{\prime}+a^{\prime} c+2 a^{\prime} c^{\prime}\right) m^{\prime}+a c+a c^{\prime}+a^{\prime} c+a^{\prime} c^{\prime}}$

$=\left(m-m^{\prime}\right) \frac{-2 a c m m^{\prime}+2\left(\frac{\gamma^{\prime}-\gamma}{m-m^{\prime}}\right) m^{2} m^{\prime 2}+2 a c+a c^{\prime}+a^{\prime} c+2 a^{\prime} c^{\prime}-2\left(\frac{\gamma m-\gamma^{\prime} m^{\prime}}{m-m^{\prime}}\right) m^{\prime}}{(a c+2 a c) m^{\prime 2}+\left(2 a c+3 a c^{\prime}+a^{\prime} c+2 a^{\prime} c^{\prime}\right) m^{\prime}+a c+a c^{\prime}+a^{\prime} c+a^{\prime} c^{\prime}}$

$m=m^{\prime} \Rightarrow \beta=1 \Rightarrow x=\frac{(1-\beta)\left(p_{1}+p_{2}\right)}{4}=0$, it is impossible

But $\frac{2}{2 k^{\prime}+1}=\frac{-2 a c k k^{\prime}+2 a c+a c^{\prime}+a^{\prime} c+2 a^{\prime} c^{\prime}-2 \delta k k^{\prime}}{\left(a c+2 a c^{\prime}\right) k^{\prime 2}+\left(2 a c+3 a c^{\prime}+a^{\prime} c+2 a^{\prime} c^{\prime}\right) k^{\prime}+a c+a c^{\prime}+a^{\prime} c+a^{\prime} c^{\prime}}$

For $\left(a, a^{\prime}, c, c^{\prime}\right) \mid a c^{\prime}=\delta k^{2}=\gamma m^{2}, \quad a^{\prime} c=\delta k^{\prime 2}=\gamma^{\prime} m^{\prime 2}$

$\Rightarrow \frac{2}{2 m^{\prime}+1}=\frac{-2 a c m m^{\prime}+2 a c+a c^{\prime}+a^{\prime} c+2 a^{\prime} c^{\prime}-2 \gamma m m^{\prime}}{\left(a c+2 a c^{\prime}\right) m^{\prime 2}+\left(2 a c+3 a c^{\prime}+a^{\prime} c+2 a^{\prime} c^{\prime}\right) m^{\prime}+a c+a c^{\prime}+a^{\prime} c+a^{\prime} c^{\prime}}$

$=\frac{-2 a c m m^{\prime}+2 a c+a c^{\prime}+a^{\prime} c+2 a^{\prime} c^{\prime}-2 \gamma^{\prime} m m^{\prime}}{\left(a c+2 a c^{\prime}\right) m^{\prime 2}+\left(2 a c+3 a c^{\prime}+a^{\prime} c+2 a^{\prime} c^{\prime}\right) m^{\prime}+a c+a c^{\prime}+a^{\prime} c+a^{\prime} c^{\prime}}$

$\Rightarrow \frac{2}{2 m^{\prime}+1}=\frac{-2 a c m m^{\prime}+2\left(\frac{\gamma^{\prime}-\gamma}{m-m^{\prime}}\right) m^{2} m^{\prime 2}+2 a c+a c^{\prime}+a^{\prime} c+2 a^{\prime} c^{\prime}-\left(\frac{\gamma m-\gamma^{\prime} m^{\prime}}{m-m^{\prime}}\right) m m^{\prime}}{(a c+2 a c) m^{\prime 2}+\left(2 a c+3 a c^{\prime}+a^{\prime} c+2 a^{\prime} c^{\prime}\right) m^{\prime}+a c}$
$\left.=\frac{-2 a c m m^{\prime}+2 a c+a c^{\prime}+a^{\prime} c+2 a^{\prime} c^{\prime}-2 \gamma m m^{\prime}}{\left(a c+2 a c^{\prime}\right) m^{\prime 2}+\left(2 a c+3 a c^{\prime}+a^{\prime} c+2 a^{\prime} c^{\prime}\right) m^{\prime}+a c+a c} a^{\prime} c\right)$

$=\frac{-2 a c m m^{\prime}+2\left(\frac{\gamma-\gamma}{m-m^{\prime}}\right) m^{2} m^{\prime 2}+2 a c+a c^{\prime}+a^{\prime} c+2 a^{\prime} c^{\prime}-\gamma m^{\prime}}{(a c+2 a c) k^{\prime 2}+\left(2 a c+3 c^{\prime}+a^{\prime} c+2 a^{\prime}\right)^{\prime}}$

$\Rightarrow \gamma=\gamma^{\prime} \Rightarrow a c^{\prime}=\delta k^{2}=\gamma m^{2}, a^{\prime} c=\delta \quad=\gamma^{\prime} n=\gamma m^{\prime 2}$

$\Rightarrow \frac{\delta}{\gamma}=\frac{m^{2}}{k^{2}}=\frac{m^{\prime 2}}{k^{\prime 2}} \Rightarrow \frac{k^{2}}{k^{\prime 2}}=\left(2 k+1=\alpha^{2}=\frac{m}{m}=(2 m+1)^{2}=\beta^{2}\right.$

If $\alpha=-\beta \Rightarrow(\beta-\alpha) p_{1}=p_{1}=(-2-\alpha-\beta) p_{2}=-2 p_{2} \Rightarrow \alpha=-\beta=\frac{p_{2}}{p_{1}}$

$b=-(\alpha+1) \frac{p_{1}-p_{2}}{4}<-\left(\beta+\frac{p_{1}+p_{2}}{4}=-\left(\frac{A p_{2}}{4 p_{1}}\right)\left(p_{1}-p_{2}\right)=\frac{p_{2}{ }^{2}-p_{1}^{2}}{4 p_{1}}\right.$

$\Rightarrow 4 b p_{1}=p_{2}^{2}{ }^{2} \Rightarrow\left(p_{1}\right) p_{1}=\rho_{2}^{2} \Rightarrow 4 b+p_{1}=\frac{p_{2}{ }^{2}}{p_{1}}$ and it is impossible because $p_{1}$ and $p_{2}$ are primes

and $=\frac{p}{2}$ can not be an integer.

$\Rightarrow \alpha-\beta=0 \quad \beta-\alpha) p_{1}=(-2-\alpha-\beta) p_{2}=-2(1+\alpha) p_{2}=-2(1+\beta) p_{2}=0 \Rightarrow \alpha=\beta=-1$

$\Rightarrow b=-\frac{(\alpha+1)}{4}\left(p_{1}-p_{2}\right)=-\frac{(\beta+1)}{4}\left(p_{1}+p_{2}\right)=0$

$\Rightarrow x=\frac{p_{1}+p_{2}}{2}, y=\frac{p_{1}-p_{2}}{2}$

Another proof : let $\mathrm{u}, \mathrm{u}^{\prime}, \mathrm{v}, \mathrm{v}^{\prime}$ verifying

$\left(\frac{1}{u p_{1}-v p_{2}}-\frac{1}{u^{\prime} p_{1}+v^{\prime} p_{2}}\right) p_{1}=\left(\frac{1}{u p_{1}-v p_{2}}+\frac{1}{u^{\prime} p_{1}+v^{\prime} p_{2}}\right) p_{2}$

$\Rightarrow\left(u^{\prime}-u\right) p_{1}^{2}+\left(v^{\prime}+v\right) p_{1} p_{2}=\left(u+u^{\prime}\right) p_{1} p_{2}+\left(v^{\prime}-v\right) p_{2}{ }^{2}$ 
Thus

$$
\left(u^{\prime}+u-v^{\prime}-v\right) p_{1} p_{2}+\left(u-u^{\prime}\right) p_{1}^{2}=\left(v-v^{\prime}\right) p_{2}{ }^{2}
$$

We pose with a different of zero

$u^{\prime}+u-v^{\prime}-v=a(\alpha-\beta)$

$\Rightarrow\left(\left(v-v^{\prime}\right)-a(2+\alpha+\beta)\right) p_{2}{ }^{2}=\left(u-u^{\prime}\right) p_{1}{ }^{2}$

Let

$v-v^{\prime}-a(2+\alpha+\beta)=(2+\alpha+\beta)^{2}$

$u-u^{\prime}=(\alpha-\beta)^{2}$

$\Rightarrow u^{\prime} p_{1}+v^{\prime} p_{2}=\left(u-(\alpha-\beta)^{2}\right) p_{1}+\left(v-a(2+\alpha+\beta)-(2+\alpha+\beta)^{2}\right) p_{2}$

$=u p_{1}+v p_{2}-(\alpha-\beta)^{2} p_{1}-a(\alpha-\beta) p_{1}-(2+\alpha+\beta)(\alpha-\beta) p_{1}$

Let $u p_{1}=-v p_{2}$ hence

$\left(\frac{1}{u p_{1}-v p_{2}}-\frac{1}{u^{\prime} p_{1}+v^{\prime} p_{2}}\right) p_{1}=\left(\frac{1}{u p_{1}-v p_{2}}+\frac{1}{u^{\prime} p_{1}+v^{\prime} p_{2}}\right) p_{2}$

$\Rightarrow\left(\frac{1}{2 u}+\frac{1}{(\alpha-\beta)^{2}+a(\alpha-\beta)+(2+\alpha+\beta)(\alpha-\beta)}\right) p_{1}$

$=\left(\frac{1}{2 u}-\frac{1}{(\alpha-\beta)^{2}+a(\alpha-\beta)+(2+\alpha+\beta)(\alpha-\beta)}\right) p_{2}$

$\Rightarrow \frac{p_{1}-p_{2}}{2 u}=-p_{1}\left(\frac{1}{(2+\alpha+\beta)(2 \alpha+a+2)}+\frac{}{(\alpha-\beta)(2}\right.$

Let $2 u=\alpha-\beta$ hence

$\frac{1}{\alpha-\beta}-\frac{1}{\alpha+\beta+2}=-\frac{1}{(2+\alpha+\beta)(\alpha+2)}$

$\Rightarrow \frac{2 \alpha+a+3}{\alpha-\beta}=\frac{2 \alpha+a+1}{2+\alpha+\beta}$

It means $b=0$ thus

$x+y=p_{1}, x-y=p_{2}$, e prin $\mathrm{v}=\mathrm{r}$ is the primal radius. As there is the condition $\mathrm{p}_{2}<\mathrm{x}<\mathrm{p}_{1}$, there is not an infi ity of $p_{2}, p_{1}$

If $\left(x_{1}-x_{2}\right)\left(x_{1}+x_{3}\right) \quad \Rightarrow\left(x_{4}, \quad\left(x_{4}-x_{3}\right)=0\right.$

Let $\frac{x_{4}+x_{2}}{p_{1}+p_{2}}=\frac{p_{1}+p_{2}}{p_{1}+p_{2}}-1-\frac{4 b}{p_{1}} p_{2}$

$\frac{p_{1}+p_{2}}{x_{4}+r_{2}} \frac{x_{4}+x_{2}}{x_{4}+} \frac{4 b}{x+x_{2}}$

$k=-\frac{2 b}{p_{1}+p_{2}}=\frac{20}{x_{1}+x_{2}}$

$\frac{x_{4}-x_{3}}{p_{1}-p_{2}}=\frac{p_{1}-p_{2} / 4 b}{p_{1}-p_{2}}=1-\frac{4 b}{p_{1}-p_{2}}$

$\frac{p_{1}-p_{2}}{x_{4}-x_{3}}=\frac{x_{4}-x_{3}+4 b}{x_{4}-x_{3}}=1+\frac{4 b}{x_{4}-x_{3}}$

$m=-\frac{2 b}{p_{1}-p_{2}}, m^{\prime}=\frac{2 b}{x_{4}-x_{3}}$

With the same reasoning and calculus $\Rightarrow b=0$ But $b$ can not be equal to zero in all cases, it means there is an impossibility related to the fact that the conjecture is indecidable and if it is so, it is true ! Because, we would find in the case it is indecidable and false with the computer the $2 \mathrm{x}$ different of all sum of primes and it is contradictory! 
Now, if we suppose that for all $p_{2}, p_{1}$ primes, exixts $x \mid 2 x \neq p_{1}-p_{2}$

$x=\frac{p_{1}-p_{2}}{2}+b, y=\frac{p_{1}+p_{2}}{2}+b$,

with the same reasoning, the same calculus but replacing $x$ by $y$ and $y$ by $x$, we prove that $\mathrm{b}=0$, which means that for all positive integer $x$, exists $p_{1}, p_{2}$ for which $x=\frac{p_{1}-p_{2}}{2}$, if we pose $y=\frac{p_{1}+p_{2}}{2}, x+y=p_{1}, y-x=p_{2} y$ is the primal radius. As there is no condition on $x, y, p_{1}, p_{2}$ there is an infinity of couples of primes $\left(p_{1,} p_{2},\right)$ For $\mathrm{x}=1, p_{1}$, and $p_{2}$ are twin primes. Let us prove it. Let us suppose that exists an integer $\mathrm{x} \geq 0$ for which $2 x$ is never the difference of two primes, then for all $p_{1}$ and $p_{2}$ primes, or $2 x=p_{1}-p_{2}+2 b_{p_{1}, p_{2}}=p_{1}-p_{2}+2 b$, then $x=\frac{p_{1}-p_{2}}{2}+b$.

But for all $p_{1,} p_{2}$ exists $y$, for which $y=\frac{p_{1}+p_{2}}{2}+b$

Let

$x_{1}=p_{1}+2 b, x_{2}=p_{2}-2 b, x_{3}=p_{2}+2 b, x_{4}=p_{1}-2 b$

We deduce that

$y=\frac{p_{1}+p_{2}}{2}+b=\frac{p_{1}+x_{2}}{2}+2 b=\frac{x_{1}+p_{2}}{2}=\frac{x_{1}+x_{2}}{2}+b$

$=\frac{p_{1}+x_{3}}{2}=\frac{x_{1}+x_{3}}{2}-b=\frac{x_{4}+x_{3}}{2}+b=\frac{x_{4}+x_{2}}{2}+3 b$

$x=\frac{p_{1}-p_{2}}{2}+b=\frac{p_{1}-x_{2}}{2}=\frac{x_{1}-p_{2}}{2}=\frac{x_{1}-x_{2}}{2}-b$

$=\frac{p_{1}-x_{3}}{2}+2 b=\frac{x_{1}-x_{3}}{2}+b=\frac{x_{4}-x_{3}}{2}+3 b=\frac{x_{4}-x_{2}}{2}+b$

$x_{1}+x_{2}=p_{1}+p_{2}$

$x_{1}-x_{3}=p_{1}-p_{2}$

\section{Lemma 2}

The following formula

$y=\frac{p_{1}+p_{2}}{2}+b \quad p_{1}+x_{2}-2 b=\frac{x_{1}-p_{2}}{2}=\frac{x_{1}+x_{2}}{2}+b$

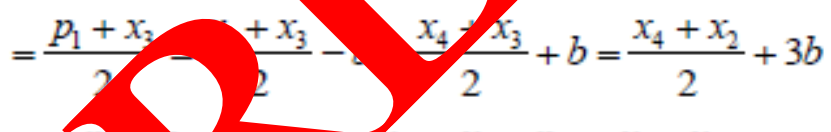

$x=\frac{p_{1}}{2}+o-\frac{x_{2}}{2}=\frac{x_{1}-p_{2}}{2}=\frac{x_{1}-x_{2}}{2}-b$

$=\frac{p_{1}-x_{3}}{2}+2 y=\frac{x_{1}-x_{3}}{2}+b=\frac{x_{4}-x_{3}}{2}+3 b=\frac{x_{4}-x_{2}}{2}+b$

Imply that exist $p_{1}$ and $p_{2}$ prime numbers for which $b=0$

\section{Proof of lemma 2}

If $x$ is prime $0=x-x$ is the sum of two primes, then $p_{1}-p_{2} \neq 0$

We will suppose firstly that $\left(x_{1}-x_{2}\right)\left(x_{1}+x_{3}\right) \neq 0$ 
Let

$$
\begin{aligned}
& \frac{x_{1}-x_{2}}{p_{1}-p_{2}}=\frac{p_{1}-p_{2}+4 b}{p_{1}-p_{2}}=1+\frac{4 b}{p_{1}-p_{2}} \\
& \frac{p_{1}-p_{2}}{x_{1}-x_{2}}=\frac{x_{1}-x_{2}-4 b}{x_{1}-x_{2}}=1-\frac{4 b}{x_{1}-x_{2}}
\end{aligned}
$$

We pose $k=\frac{2 b}{p_{1}-p_{2}}, k^{\prime}=-\frac{2 b}{x_{1}-x_{2}}$

$k k^{\prime}=0 \Rightarrow b=0$, we have supposed $k k^{\prime} \neq 0$

$\forall(x, y) ; \exists \varphi \mid y=\varphi x$

$x+y=(\varphi+1) x=x_{1} \neq 0, y-x=(\varphi-1) x=p_{2} \neq 0$

$\forall(k, k) ; \exists \alpha \mid k=\alpha k^{\prime}$

$\Rightarrow \frac{2 b}{p_{1}-p_{2}}=-\alpha \frac{2 b}{x_{1}-x_{2}}$

$\Rightarrow x_{1}-x_{2}=-\alpha\left(p_{1}-p_{2}\right) \Rightarrow x_{1}-x_{2}-p_{1}+p_{2}=4 b=-(\alpha+1)\left(p_{1}-p_{2}\right)$

$\Rightarrow b=-\frac{\alpha+1}{4}\left(p_{1}-p_{2}\right)$

$\Rightarrow y=\frac{p_{1}+p_{2}}{2}+b=\frac{p_{1}+p_{2}}{2}-\frac{\alpha+1}{4}\left(p_{1}-p_{2}\right)=\frac{(1-\alpha) p_{1}+(3+\alpha) p_{2}}{4}=\frac{\varphi}{\varphi-1}$ $x=\frac{p_{1}-p_{2}}{2}+b=\frac{p_{1}-p_{2}}{2}-\frac{\alpha+1}{4}\left(p_{1}-p_{2}\right)=\frac{(1-\alpha)\left(p_{1}-p_{2}\right)}{4}=\frac{1}{\varphi-1} p_{\gamma}$

Let

$$
\begin{aligned}
& \frac{x_{1}+x_{3}}{p_{1}+p_{2}}=\frac{p_{1}+p_{2}+4 b}{p_{1}+p_{2}}=1+\frac{4 b}{p_{1}+p_{2}} \\
& \frac{p_{1}+p_{2}}{x_{1}+x_{3}}=\frac{x_{1}+x_{3}-4 b}{x_{1}+x_{3}}=1-\frac{4 b}{x_{1}+x_{3}}
\end{aligned}
$$

$$
\begin{aligned}
& \text { We pose } m=\frac{2 b}{p_{1}+p_{2}}, m^{\prime}=0 \\
& m m^{\prime}=0 \Rightarrow b=0 \text {, we } \\
& \forall\left(m, m^{\prime}\right) ; \beta \mid m=m^{\prime} \\
& \Rightarrow \frac{2 b}{p_{1}+p_{2}}=-\beta \frac{x_{1}}{x_{1}+} \\
& \left.\Rightarrow x_{1}+=-p_{1}+p_{2}\right) \\
& \Rightarrow b=-1, x_{3}-p_{1}-p_{2}=4 b=-(\beta+1)\left(p_{1}+p_{2}\right) \\
& \Rightarrow y=\frac{p_{1}+\lambda}{2} b=\frac{p_{1}+p_{2}}{2}-\frac{\beta+1}{4}\left(p_{1}+p_{2}\right)=\frac{(1-\beta)\left(p_{1}+p_{2}\right)}{4}=\frac{\varphi}{\varphi-1} p_{2} \\
& x=\frac{p_{1}-p_{2}}{2}+b=\frac{p_{1}-p_{2}}{2}-\frac{\beta+1}{4}\left(p_{1}+p_{2}\right)=\frac{(1-\beta) p_{1}-(\beta+3) p_{2}}{4}=\frac{1}{\varphi-1} p_{2} \\
& b=-\frac{\alpha+1}{4}\left(p_{1}-p_{2}\right)=-\frac{\beta+1}{4}\left(p_{1}+p_{2}\right) \Rightarrow(\beta-\alpha) p_{1}=(-2-\alpha-\beta) p_{2}
\end{aligned}
$$


But : $(2 k+1)\left(2 k^{\prime}+1\right)=1 \Rightarrow 2 k k^{\prime}+k+k^{\prime}=0 \Rightarrow(2 k+1) k^{\prime}=-k$

$2 k+1=\frac{-k}{k^{\prime}}=\frac{k+1}{k^{\prime}+1}=\frac{a(k+1)+a^{\prime}(2 k+1)}{a\left(k^{\prime}+1\right)+a^{\prime}}$

$2 k^{\prime}+1=\frac{-k^{\prime}}{k}=\frac{k^{\prime}+1}{k+1}=\frac{c\left(k^{\prime}+1\right)+c^{\prime}\left(2 k^{\prime}+1\right)}{c(k+1)+c^{\prime}}, \forall\left(a, a^{\prime}, c, c^{\prime}\right)$

$\frac{2 k+1}{2 k^{\prime}+1}=\frac{\left(\left(a+2 a^{\prime}\right) k+a+a^{\prime}\right)\left(c k+c+c^{\prime}\right)}{\left(\left(c+2 c^{\prime}\right) k^{\prime}+c+c^{\prime}\right)\left(a k^{\prime}+a+a^{\prime}\right)}$

$=\frac{\left(a c+2 a^{\prime} c\right) k^{2}+\left(2 a c+a c^{\prime}+3 a^{\prime} c+2 a^{\prime} c^{\prime}\right) k+a c+a c^{\prime}+a^{\prime} c+a^{\prime} c^{\prime}}{(a c+2 a c) k^{\prime 2}+\left(2 a c+3 a c^{\prime}+a^{\prime} c+2 a^{\prime} c^{\prime}\right) k^{\prime}+a c+a c^{\prime}+a^{\prime} c+a^{\prime} c^{\prime}}$

$\Rightarrow \frac{2 k+1}{2 k^{\prime}+1}-1=\left(k-k^{\prime}\right) \frac{2}{2 k^{\prime}+1}$

$=\frac{a c\left(k^{2}-k^{\prime 2}\right)+2 a^{\prime} c k^{2}-2 a c^{\prime} k^{\prime 2}+\left(2 a c+a c^{\prime}+a^{\prime} c+2 a^{\prime} c^{\prime}\right)\left(k-k^{\prime}\right)+2 a^{\prime} c k-2 a c^{\prime} k^{\prime}}{(a c+2 a c) k^{\prime 2}+\left(2 a c+3 a c^{\prime}+a^{\prime} c+2 a^{\prime} c^{\prime}\right) k^{\prime}+a c+a c^{\prime}+a^{\prime} c+a^{\prime} c^{\prime}}$

$\forall\left(a, a^{\prime}, c, c^{\prime}\right)$, particularly $\left(a, a^{\prime}, c, c^{\prime}\right) \mid a c^{\prime}=\delta k^{2}, a^{\prime} c=\delta k^{\prime 2}$

$\Rightarrow\left(k-k^{\prime}\right) \frac{2}{2 k^{\prime}+1}$

$=\frac{a c\left(k+k^{\prime}\right)\left(k-k^{\prime}\right)+\left(2 a c+a c^{\prime}+a^{\prime} c+2 a^{\prime} c^{\prime}\right)(k-k)+2 \delta k k^{\prime 2}-2 \delta k^{2} k^{\prime}}{\left(a c+2 a c^{\prime}\right) k^{\prime 2}+\left(2 a c+3 a c^{\prime}+a^{\prime} c+2 a^{\prime} c^{\prime}\right) k^{\prime}+a c+a c^{\prime}+a^{\prime} c+a^{\prime} c^{\prime}}$

$=\left(k-k^{\prime}\right) \frac{-2 a c k k^{\prime}+2 a c+a c^{\prime}+a^{\prime} c+2 a^{\prime} c^{\prime}-2 \delta k k^{\prime}}{\left(a c+2 a c^{\prime}\right) k^{\prime 2}+\left(2 a c+3 a c^{\prime}+a^{\prime} c+2 a^{\prime} c^{\prime}\right) k^{\prime}+a c+a c^{\prime}+a^{\prime}+a^{\prime} c^{\prime}}$

$k=k^{\prime} \Rightarrow \alpha=1 \Rightarrow y=\frac{(1-\alpha) p_{1}+(3+\alpha) p_{2}}{4}=p_{2}$ it is impossi

$k-k^{\prime} \neq 0 \Rightarrow \frac{2}{2 k^{\prime}+1}=\frac{-2 a c k k^{\prime}+2 a c+a c^{\prime}+a^{\prime} c+2 d}{\left(a c+2 a c^{\prime}\right) k^{\prime 2}+\left(2 a c+3 a c^{\prime}+a\right.}$

Also $(2 m+1)\left(2 m^{\prime}+1\right)=1 \Rightarrow 2 m m^{\prime}+m+m^{\prime}=0 \Rightarrow\left(2 z^{\prime}\right) m, m$

$2 m+1=\frac{-m}{m^{\prime}}=\frac{m+1}{m^{\prime}+1}=\frac{a(m+1)+a^{\prime}(2 m+1)}{a\left(m^{\prime}+1\right)+}$

$2 m^{\prime}+1==\frac{-m^{\prime}}{m}=\frac{m^{\prime}+1}{m+1}=\frac{c\left(m^{\prime}+1\right)}{m+1)+c}$

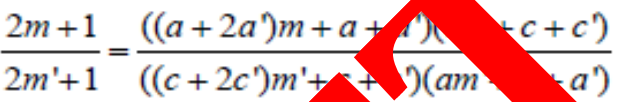

$=\frac{\left(a c+2 a^{\prime} c\right) m^{2}+2 a c+\left(c^{\prime}+3 a^{\prime} c+2, c^{\prime}\right) m+a c+a c^{\prime}+a^{\prime} c+a^{\prime} c^{\prime}}{(a c+2 a c) m^{\prime}}$

$\Rightarrow \frac{2 m+1}{2 m^{\prime}}(m-1) 2$

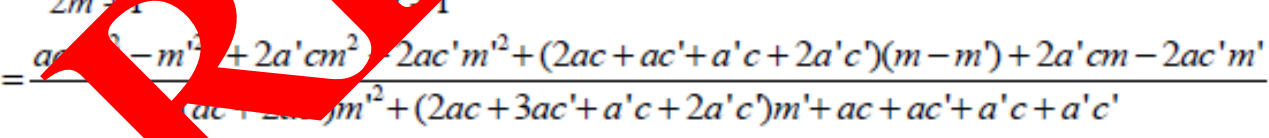

$\forall\left(a, a^{\prime}, c, c^{\prime}\right)$ ayticularly $\left(a, a^{\prime}, c, c^{\prime}\right) \mid a c^{\prime}=\delta k^{2}=\gamma m^{2}, a^{\prime} c=\delta k^{\prime 2}=\gamma^{\prime} m^{\prime 2}$

$\Rightarrow\left(m-m^{\prime}\right) \frac{2}{2 m^{\prime}+1}$

$=\frac{a c\left(m+m^{\prime}\right)\left(m-m^{\prime}\right)+2\left(\gamma^{\prime}-\gamma\right) m^{2} m^{\prime 2}+\left(2 a c+a c^{\prime}+a^{\prime} c+2 a^{\prime} c^{\prime}\right)\left(m-m^{\prime}\right)+2 \gamma^{\prime} m m^{\prime 2}-2 \gamma m^{2} m^{\prime}}{\left(a c+2 a c^{\prime}\right) m^{\prime 2}+\left(2 a c+3 a c^{\prime}+a^{\prime} c+2 a^{\prime} c^{\prime}\right) m^{\prime}+a c+a c^{\prime}+a^{\prime} c+a^{\prime} c^{\prime}}$

$=\left(m-m^{\prime}\right) \frac{-2 a c m m^{\prime}+2\left(\frac{\gamma^{\prime}-\gamma}{m-m^{\prime}}\right) m^{2} m^{\prime 2}+2 a c+a c^{\prime}+a^{\prime} c+2 a^{\prime} c^{\prime}-2\left(\frac{\gamma m-\gamma^{\prime} m^{\prime}}{m-m^{\prime}}\right) m m^{\prime}}{\left(a c+2 a c^{\prime}\right) m^{\prime 2}+\left(2 a c+3 a c^{\prime}+a^{\prime} c+2 a^{\prime} c^{\prime}\right) m^{\prime}+a c+a c^{\prime}+a^{\prime} c+a^{\prime} c^{\prime}}$

$m=m^{\prime} \Rightarrow \beta=1 \Rightarrow y=\frac{(1-\beta)\left(p_{1}+p_{2}\right)}{4}=0$, it is impossible 
But $\frac{2}{2 k^{\prime}+1}=\frac{-2 a c k k^{\prime}+2 a c+a c^{\prime}+a^{\prime} c+2 a^{\prime} c^{\prime}-2 \delta k k^{\prime}}{\left(a c+2 a c^{\prime}\right) k^{\prime 2}+\left(2 a c+3 a c^{\prime}+a^{\prime} c+2 a^{\prime} c^{\prime}\right) k^{\prime}+a c+a c^{\prime}+a^{\prime} c+a^{\prime} c^{\prime}}$

For $\left(a, a^{\prime}, c, c^{\prime}\right) \mid a c^{\prime}=\delta k^{2}=\gamma m^{2}, \quad a^{\prime} c=\delta k^{\prime 2}=\gamma^{\prime} m^{\prime 2}$

$\Rightarrow \frac{2}{2 m^{\prime}+1}=\frac{-2 a c m m^{\prime}+2 a c+a c^{\prime}+a^{\prime} c+2 a^{\prime} c^{\prime}-2 \gamma m m^{\prime}}{\left(a c+2 a c^{\prime}\right) m^{\prime 2}+\left(2 a c+3 a c^{\prime}+a^{\prime} c+2 a^{\prime} c^{\prime}\right) m^{\prime}+a c+a c^{\prime}+a^{\prime} c+a^{\prime} c^{\prime}}$

$=\frac{-2 a c m m^{\prime}+2 a c+a c^{\prime}+a^{\prime} c+2 a^{\prime} c^{\prime}-2 \gamma^{\prime} m m^{\prime}}{\left(a c+2 a c^{\prime}\right) m^{\prime 2}+\left(2 a c+3 a c^{\prime}+a^{\prime} c+2 a^{\prime} c^{\prime}\right) m^{\prime}+a c+a c^{\prime}+a^{\prime} c+a^{\prime} c^{\prime}}$

$\Rightarrow \frac{2}{2 m^{\prime}+1}=\frac{-2 a c m m^{\prime}+2\left(\frac{\gamma^{\prime}-\gamma}{m-m^{\prime}}\right) m^{2} m^{\prime 2}+2 a c+a c^{\prime}+a^{\prime} c+2 a^{\prime} c^{\prime}-2\left(\frac{\gamma m-\gamma^{\prime} m^{\prime}}{m-m^{\prime}}\right) m m^{\prime}}{(a c+2 a c) m^{\prime 2}+\left(2 a c+3 a c^{\prime}+a^{\prime} c+2 a^{\prime} c^{\prime}\right) m^{\prime}+a c+a c^{\prime}+a^{\prime} c+a^{\prime} c^{\prime}}$

$=\frac{-2 a c m m^{\prime}+2 a c+a c^{\prime}+a^{\prime} c+2 a^{\prime} c^{\prime}-2 \gamma m m^{\prime}}{\left(a c+2 a c^{\prime}\right) m^{\prime 2}+\left(2 a c+3 a c^{\prime}+a^{\prime} c+2 a^{\prime} c^{\prime}\right) m^{\prime}+a c+a c^{\prime}+a^{\prime} c+a^{\prime} c^{\prime}}$

$=\frac{-2 a c m m^{\prime}+2\left(\frac{\gamma-\gamma}{m-m^{\prime}}\right) m^{2} m^{\prime 2}+2 a c+a c^{\prime}+a^{\prime} c+2 a^{\prime} c^{\prime}-2 \gamma m m^{\prime}}{\left(a c+2 a c^{\prime}\right) k^{\prime 2}+\left(2 a c+3 a c^{\prime}+a^{\prime} c+2 a^{\prime} c^{\prime}\right) k^{\prime}+a c+a c^{\prime}+a^{\prime} c+a^{\prime} c^{\prime}}$

$\Rightarrow \gamma=\gamma^{\prime} \Rightarrow a c^{\prime}=\delta k^{2}=\gamma m^{2}, a^{\prime} c=\delta k^{\prime 2}=\gamma^{\prime} m^{\prime 2}=\gamma m^{\prime 2}$

$\Rightarrow \frac{\delta}{\gamma}=\frac{m^{2}}{k^{2}}=\frac{m^{\prime 2}}{k^{\prime 2}} \Rightarrow \frac{k^{2}}{k^{\prime 2}}=(2 k+1)^{2}=\alpha^{2}=\frac{m^{2}}{m^{\prime 2}}=(2 m+1)^{2}=\beta^{2}$

If $\alpha=-\beta \Rightarrow(\beta-\alpha) p_{1}=-2 \alpha p_{1}=2 \beta p_{1}=(-2-\alpha-\beta) p_{2}=-2 p_{2} \Rightarrow \alpha=\alpha \frac{p_{2}}{p_{1}}$

$b=-(\alpha+1) \frac{p_{1}-p_{2}}{4}=-(\beta+1) \frac{p_{1}+p_{2}}{4}=-\left(\frac{p_{1}+p_{2}}{4 p_{1}}\right)\left(p_{1}-p_{2}\right)=\frac{p_{2}{ }^{2}-}{4 p}$

$\Rightarrow 4 b p_{1}=p_{2}{ }^{2}-p_{1}{ }^{2} \Rightarrow\left(4 b+p_{1}\right) p_{1}=p_{2}{ }^{2} \Rightarrow 4 b+p_{1}=\frac{p_{2}{ }^{2}}{p_{1}}$ and it is ossibl because $p_{1}$ and $p_{2}$ are primes and $4 b+p_{1}=\frac{p_{2}^{2}}{p_{1}}$ can not be an integer.

$\left.\left.\Rightarrow \alpha-\beta=0 \Rightarrow(\beta-\alpha) p_{1}=(-2-\alpha-\beta) p_{2}=>2\right) p_{2}=-2+\beta\right) p_{2}=0 \Rightarrow \alpha=\beta=-1$

$\Rightarrow b=-\frac{(\alpha+1)}{4}\left(p_{1}-p_{2}\right)=-\frac{(\beta+1)}{4}\left(p_{1}\right.$

$\Rightarrow y=\frac{p_{1}+p_{2}}{2}, x=\frac{p_{1}-p_{2}}{2}$

Another proof : let u, u' v' ving

$\left(\frac{1}{u p_{1}-v p_{2}}-\frac{1}{u^{\prime} \gamma+v^{\prime} \hat{s}}\right) p_{1}=\left(\frac{1}{u p_{1}-v p_{2}}+\frac{1}{u^{\prime} p_{1}+v^{\prime} p_{2}}\right) p_{2}$

$\Rightarrow\left(u^{\prime}-u\right) p_{1}^{2}+\left(v \quad\left\langle p_{1} p_{2}=\left(u+u^{\prime}\right) p_{1} p_{2}+\left(v^{\prime}-v\right) p_{2}^{2}\right.\right.$

Thus

$\left(u^{\prime}+v^{\prime}-v\right) p_{1} p_{2}-\left(u-u^{\prime}\right) p_{1}{ }^{2}=\left(v-v^{\prime}\right) p_{2}{ }^{2}$

We pose a dinterent of zero

$u^{\prime}+u-v^{\prime}-\mathrm{g}(\alpha-\beta)$

$\Rightarrow\left(\left(v-v^{\prime}\right)-a(2+\alpha+\beta)\right) p_{2}{ }^{2}=\left(u-u^{\prime}\right) p_{1}{ }^{2}$

Let

$v-v^{\prime}-a(2+\alpha+\beta)=(2+\alpha+\beta)^{2}$

$u-u^{\prime}=(\alpha-\beta)^{2}$

$\Rightarrow u^{\prime} p_{1}+v^{\prime} p_{2}=\left(u-(\alpha-\beta)^{2}\right) p_{1}+\left(v-a(2+\alpha+\beta)-(2+\alpha+\beta)^{2}\right) p_{2}$

$=u p_{1}+v p_{2}-(\alpha-\beta)^{2} p_{1}-a(\alpha-\beta) p_{1}-(2+\alpha+\beta)(\alpha-\beta) p_{1}$ 
Let $u p_{1}=-v p_{2}$ hence

$\left(\frac{1}{u p_{1}-v p_{2}}-\frac{1}{u^{\prime} p_{1}+v^{\prime} p_{2}}\right) p_{1}=\left(\frac{1}{u p_{1}-v p_{2}}+\frac{1}{u^{\prime} p_{1}+v^{\prime} p_{2}}\right) p_{2}$

$\Rightarrow\left(\frac{1}{2 u}+\frac{1}{(\alpha-\beta)^{2}+a(\alpha-\beta)+(2+\alpha+\beta)(\alpha-\beta)}\right) p_{1}$

$=\left(\frac{1}{2 u}-\frac{1}{(\alpha-\beta)^{2}+a(\alpha-\beta)+(2+\alpha+\beta)(\alpha-\beta)}\right) p_{2}$

$\Rightarrow \frac{p_{1}-p_{2}}{2 u}=-p_{1}\left(\frac{1}{(2+\alpha+\beta)(2 \alpha+a+2)}+\frac{1}{(\alpha-\beta)(2 \alpha+a+2)}\right)$

Let $2 u=\alpha-\beta$ hence

$\frac{1}{\alpha-\beta}-\frac{1}{\alpha+\beta+2}=-\frac{1}{(2+\alpha+\beta)(2 \alpha+a+2)}-\frac{1}{(\alpha-\beta)(2 \alpha+a+2)}$

$\Rightarrow \frac{2 \alpha+a+3}{\alpha-\beta}=\frac{2 \alpha+a+1}{2+\alpha+\beta} ; \forall a \Rightarrow \alpha=\beta=-1$

It means $\mathrm{b}=0$ thus

$x+y=p_{1}, y-x=p_{2}$ are primes $y=r$ is the primal radius. As ther is condition, nere is an infinity of $p_{1,} p_{2}$.

If $\left(x_{1}-x_{2}\right)\left(x_{1}+x_{3}\right)=0 \Rightarrow\left(x_{4}+x_{2}\right)\left(x_{4}-x_{3}\right) \neq 0$

Let $\frac{x_{4}+x_{2}}{p_{1}+p_{2}}=\frac{p_{1}+p_{2}-4 b}{p_{1}+p_{2}}=1-\frac{4 b}{p_{1}+p_{2}}$

$\frac{p_{1}+p_{2}}{x_{4}+x_{2}}=\frac{x_{4}+x_{2}+4 b}{x_{4}+x_{2}}=1+\frac{4 b}{x_{4}+x_{2}}$

$k=-\frac{2 b}{p_{1}+p_{2}}, k^{\prime}=\frac{2 b}{x_{4}+x_{2}}$

$\frac{x_{4}-x_{3}}{p_{1}-p_{2}}=\frac{p_{1}-p_{2}-4 b}{p_{1}-p_{2}}=1-\frac{4 b}{p_{1}-p_{2}}$

$\frac{p_{1}-p_{2}}{x_{4}-x_{3}}=\frac{x_{4}-x_{3}+4 b}{x_{4}-x_{3}}=1+\frac{4 b}{x_{4}-}$

$m=-\frac{2 b}{p_{1}-p_{2}}, m^{\prime}=\frac{2 b}{x_{4}}$

With the same calulus and reaso $g$, it implies that $b=0$. But $b$ can not be equal to zero in all cases, it means re is immossibility related to the fact that the conjecture is indecidable and if it is so, it is true ! B se, we ould find in the case it is indecidable and false with the computer the $2 \mathrm{x}$ differe all su $\mathrm{pr}$. nes and it is contradictory!

For ${ }^{2}=p_{1}-p_{2}$ a diffe nce of an infinity of couples of primes. There is an infinity of consecutive primes. A for an 2104 exists $p_{1}=p_{2}+2, p_{2}$ primes for which $2 x=p_{1}+p_{2}$

\section{Conclusion}

The notion of the primal radius as defined in this study allows to confirm that for all integer $\mathrm{x} \geq 3$ exists a number $\mathrm{r}>0$ for which $\mathrm{x}+\mathrm{r}$ and $\mathrm{x}-\mathrm{r}$ are primes and that for all integer $\mathrm{x} \geq 0$ exists a number $\mathrm{r}>0$ for which $\mathrm{x}+\mathrm{r}$ and $\mathrm{r}-\mathrm{x}$ are primes and that exists an infinty of such primes. $\mathrm{r}$ is called the primal radius The corrolary is the proof of the Goldbach conjecture and de Polignac conjecture which stipulate, the first that an even number is always the sum of two prime numbers, the second that an even number is always the difference between two primes and that there is an infinity of such couples of primes. Another corollary is the proof of the twin primes conjecture which stipulates that there is an infinity of consecutive primes. 


\section{References}

[1] J. R. Chen, 2002, On the representation of a larger even integer as the sum of a prime and the product of at most two primes. Sci. Sinica 16, 157-176.

[2] D. R. Heath-Brown, J. C. Puchta, 2002, Integers represented as a sum of primes and powers of two. The Asian Journal of Mathematics, 6, no. 3, pages 535-565.

[3] H.L. Montgomery, Vaughan, R. C., 1975, The exceptional set in Goldbach's problem. Collection of articles in memory of Jurii Vladimirovich Linnik. Acta Arith. 27, 353-370.

[4] J. Richstein, 2001, Verifying the goldbach conjecture up to 4. 1014, wath. mp., $70: 236,1745--1749$.

[5] L. E. Dickson, 2005, History of The Theory of Numbers, Vol1, New Yor Doyer. 